\title{
Protección cardiovascular con flavonoides. Enigma farmacocinético
}

\author{
Cardiovascular protection by flavonoids. Pharmacokinetic mystery \\ Juan Duarte ${ }^{1^{*}}$, Francisco Pérez-Vizcaíno² \\ 1. Departamento de Farmacología. Facultad de Farmacia. Universidad de Granada. \\ 2. Departamento de Farmacología. Facultad de Medicina. Universidad Complutense de Madrid.
}

\section{Artículo de revisión Review Article}

\section{Correspondencia \\ Correspondence \\ Juan Duarte}

Departamento de Farmacología.

Facultad de Farmacia. Campus Universi-

tario de Cartuja s/n. 18071. Granada.

Email: jmduarte@ugr.es.

Telf: +34958241791

Conflicto de interés Competing interest

Los autores declaran que no existe conflicto de interés.

Received: 10.10 .2015 Accepted: 12.10 .2015

\section{creative \\ commons}

LICENSE 3.0 UNPORTED.

\section{RESUMEN}

Objetivos. Los flavonoides ejercen efectos beneficiosos en la prevención de las enfermedades cardiovasculares. En esta revisión trataremos de clarificar algunas preguntas fundamentales respecto a la eficacia, mecanismo de acción y biodisponibilidad de uno de los flavonoides dietéticos más abundante, la quercetina.

Métodos. Se utilizó la base de datos de la National Library of Medicine, Washington, DC (MEDLINE: PubMed). Se recopilaron todos los estudios en animales y en humanos disponibles online desde la creación de la base de datos hasta Noviembre de 2015.

Resultados. La quercetina produce un efecto vasodilatador y antihipertensor en modelos animales y en individuos hipertensos. Es eficaz en todos los modelos de hipertensión analizados, independientemente del origen de la hipertensión, del estado del sistema renina-angiotensina, del estrés oxidativo, del óxido nítrico y de otros factores. Paradójicamente, a pesar de ejercer efectos sistémicos biológicamente demostrables, no se encuentra en el plasma tras su administración oral y sus metabolitos circulantes muestran una débil actividad in vitro. La quercetina es extensamente metabolizada en derivados metilados y glucurono- y sulfo-conjugados, que son las formas circulantes en el plasma; y glucurono-, pero no sulfoconjugados, pueden ser hidrolizados a nivel vascular, produciendo la aglicona matriz que se acumula en los tejidos. La conjugación es un proceso reversible y, al menos con respecto a los efectos vasodilatador y antihipertensivo, el ciclo de conjugación-deconjugación parece ser un requisito absoluto.

Conclusiones. Los glucurono-conjugados transportan la quercetina y su forma metilada, y liberan en los tejidos la aglicona libre, que es el efector final.

Palabra clave. Quercetina, Hipertensión, Metabolitos plasmáticos.

\section{ABSTRACT}

Objetives. Flavonoids have been proposed to exert beneficial effects in the prevention of cardiovascular diseases. In this review we try to clarify some fundamental questions regarding efficacy, mechanism of action and bioavailability of one of the most widely distributed flavonoids in the diet, quercetin.

Methods. The database of the National Library of Medicine, Washington, DC (MEDLINE PubMed) was used and all the studies in animals and humans available from inception of the database until November 2015 were collected.

Results. Quercetin exerts vasodilatory and antihypertensive effects in animal models of hypertension and hypertensive subjets. Quercetin is effective in all models of hypertension analyzed, independently of the origin of the hypertension, the status of renin-angiotensin system, oxidative stress, nitric oxide, and other factors. Paradoxically, despite exerting biologically demonstrable systemic effects, it is not found in plasma after oral administration and its circulating metabolites show weak activity in vitro. Quercetin is extensively metabolized into methylated and glucurono- and sulfo-conjugated metabolites, which are the plasma circulating forms; and glucurono-, but not sulfo-conjugates, can be hydrolyzed at the vascular level, yielding the parent aglycone which accumulates in tissues. Thus conjugation is a reversible process and, at least regarding the vasodilator and antihypertensive effects, the conjugation-deconjugation cycle appears to be an absolute requirement.

Conclusions. Glucuronidated derivatives transport quercetin and its methylated form, and deliver to the tissues the free aglycone, which is the final effector.

Keywords: Quercetin, Hypertension, Plasma Metabolites. 


\section{INTRODUCCIÓN}

Los flavonoides ejercen efectos beneficiosos en la prevención de un gran número de enfermedades, incluidas el cáncer, las enfermedades cardiovasculares y neurodegenerativas. Recientemente hemos revisado las evidencias que soportan el papel beneficioso de flavonoles sobre la enfermedad cardiovascular y las potenciales dianas moleculares implicadas. ${ }^{1}$ Más de 35.000 estudios han sido publicados con flavonoides y casi 10.000 con sólo uno de ellos: la quercetina. Sin embargo, algunas preguntas fundamentales respecto a la eficacia, mecanismo de acción y biodisponibilidad de los flavonoides están todavía sin respuesta. En esta revisión trataremos de clarificar algunas de estas cuestiones.

Los flavonoides son substancias producidas como metabolitos secundarios por las plantas, cuyo elemento estructural común es la existencia de un esqueleto de difenilpirano $\left(\mathrm{C}_{6}-\mathrm{C}_{3}-\mathrm{C}_{6}\right)$, compuesto por dos anillos de fenilos (A y B) ligados a través de un anillo (C) de pirano. ${ }^{2}$ Sobre este esqueleto pueden darse miles de substituciones, lo que origina las diferentes clases de flavonoides: flavonoles, flavonas, flavanonas, flavanoles (catequinas y proantocianidinas), antocianidinas, chalconas, auronas e isoflavonas. Los flavonoides están ampliamente distribuidos en el reino vegetal, encontrándose en cantidad variable en frutas, verduras, semillas, especias y bebidas derivadas de vegetales, como el té, las infusiones, el mosto, los zumos y el vino, por lo que entran a formar parte de nuestra dieta. ${ }^{3} \mathrm{El}$ consumo diario medio en humanos de flavonoles más flavonas se ha calculado en unos 20-25 mg, ${ }^{4}$ pero hay grandes variaciones dependiendo de los hábitos nutricionales y de las zonas geográficas.

El interés en los flavonoides presentes en la dieta ha aumentado en los últimos años debido a la publicación de varios estudios epidemiológicos que han mostrado una correlación inversa entre el consumo diario de flavonoles y flavonas, y una menor incidencia y mortalidad de cáncer y enfermedades cardiovasculares. ${ }^{3,5,6}$ El meta-análisis de siete estudios prospectivos de cohortes determinó que estar entre los individuos del tercio superior en la ingesta diaria de flavonoles se asociaba a un menor riesgo de mortalidad debida a enfermedad coronaria comparados con el tercio inferior, después de ajustar el resto de factores de riesgo y de otros componentes dietéticos. ${ }^{7}$ Recientemente se ha descrito que la mayor ingesta de frutas y verduras durante la edad joven se asocia son una menor prevalencia de enfermedad arterial coronaria después de 20 años de seguimiento. $^{8}$
La mayoría de los datos disponibles se basan en alimentos ricos en flavonoides, como el cacao, o en bebidas que los contienen en altas proporciones, como el vino, los zumos y el té; o en extractos de vino o zumos de frutas; o en algunos compuestos aislados como kaempferol, delfinidina y quercetina; lo cual hace muy difícil extrapolar resultados al comparar unos con otros. El flavonol quercetina representa el $60-75 \%$ del total de flavonoles más flavonas de la dieta, ${ }^{3}$ siendo las principales fuentes de flavonoles las cebollas (350-1200 mg/kg), la col rizada, el puerro y los tomates cherris. ${ }^{4}$

Los flavonoides muestran una amplia gama de actividades biológicas cardiovasculares (Figura 1), siendo los flavonoles los compuestos más activos dentro del grupo. Por lo tanto, los efectos beneficiosos de las dietas ricas en frutas y verduras, en la salud cardiovascular, han sido a menudo atribuidos a los flavonoides en general y más específicamente a los flavonoles. Los flavonoles son comercializados como suplementos dietéticos, ya sea como compuestos puros (quercetina), como mezclas de flavonoides o como extractos, a menudo a dosis que exceden en gran medida la ingesta dietética. Los polifenoles pueden proteger el sistema cardiovascular previniendo la oxidación de las lipoproteínas de baja densidad (LDL), la adhesión y la agregación plaquetaria y la migración y proliferación de células musculares lisas vasculares. Alternativamente, la protección vascular puede estar relacionada con una acción directa de los polifenoles sobre la función endotelial.

Figura 1. Acciones cardiovasculares de los flavonoides

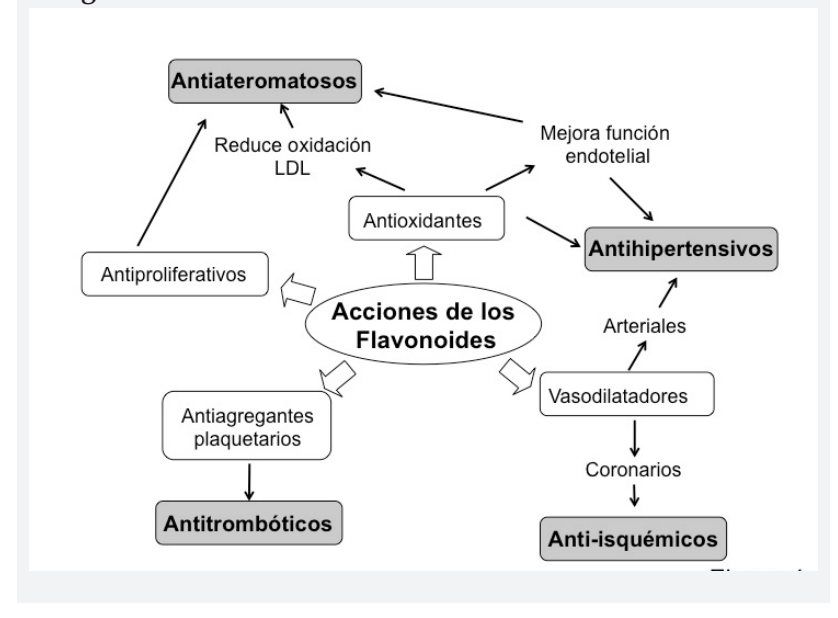

\section{MATERIALES Y MÉTODOS}

Se utilizó la base de datos de la National Library of Medicine, Washington, DC (MEDLINE: PubMed). Se recopilaron todos los estudios animales y humanos que tratan el tema de interés, escritos en Inglés, disponibles online desde la creación de la base de datos hasta Noviembre de 2015. Para 
ello, se introdujeron los siguientes términos: flavonoide o quercetina, y biodisponibilidad, e hipertensión.

\section{RESULTADOS}

\section{Flavonoides e hipertensión}

El primer informe sobre los efectos antihipertensivos de la quercetina se realizó en ratas espontáneamente hipertensas (SHR), un modelo genético de hipertensión multifactorial. ${ }^{9}$ Este estudio fue confirmado y ampliado por otros ${ }^{10-13} \mathrm{y}$ en otros modelos clásicos de hipertensión experimental. ${ }^{14-19}$ Además, la quercetina también redujo la presión arterial en modelos animales con resistencia a la insulina y síndrome metabólico tales como las ratas Zucker obesas ${ }^{20}$ y en ratas alimentadas con una dieta alta en grasas y rica en sacarosa. ${ }^{21}$ Curiosamente, la reducción de la presión arterial es de larga duración, permaneciendo por lo menos después de 48 horas de la interrupción del tratamiento. Sorprendentemente, la quercetina fue eficaz en todos los modelos de hipertensión analizados, independientemente del origen de la hipertensión, del estado del sistema renina-angiotensina, del estrés oxidativo, del óxido nítrico y de otros factores. Sin embargo, la quercetina no ejerce efectos hipotensores, es decir, no tiene ningún efecto en los animales normotensos.

De acuerdo con la llamada «hipótesis de Barker», fuertemente apoyada por los estudios epidemiológicos en humanos y animales, muchas enfermedades crónicas en adultos, incluyendo la diabetes tipo 2, la obesidad y la hipertensión, pueden tener su origen en la vida del feto debido a los cambios en la programación genética. ${ }^{22}$ Así, los descendientes adultos de las ratas o ratones alimentados con una dieta alta en grasas durante el embarazo mostraron hiperglucemia adulta, resistencia a la insulina, obesidad e hipertensión, a pesar de ser alimentados con una dieta estándar durante toda la vida postnatal. ${ }^{23}$ Recientemente, un estudio muy interesante ${ }^{24}$ mostró que estos efectos se redujeron en los ratones alimentados con dieta suplementada con quercetina durante el embarazo. Estos resultados sugieren que la quercetina puede evitar la programación epigenética durante la vida prenatal.

Una presión arterial elevada mantenida en el tiempo es uno de los determinantes más poderosos en el desarrollo de las enfermedades cardíacas, vasculares y renales. La mayoría de los beneficios del tratamiento antihipertensivo en el daño de órganos diana son el resultado de la bajada de la presión arterial per se y son, en gran medida, independientes del tipo de fármaco empleado. ${ }^{25}$ La quercetina ha demostrado una reducción de la hipertrofia ventricular izquierda y efectos protectores sobre la estructura y la función renal en los distintos modelos animales de hipertensión estudiados.
También se ha demostrado un efecto protector sobre la función endotelial en estos modelos animales.

Recientemente se han publicado dos ensayos aleatorizados, a doble ciego, controlados con placebo, ensayos clínicos cruzados, que analizan los efectos de la quercetina en la presión arterial. En el estudio de Edwards et al., ${ }^{26}$ los pacientes con hipertensión estadio 1 tuvieron una reducción en la presión arterial sistólica, diastólica y media después del tratamiento con quercetina, pero el flavonol no tuvo efectos significativos sobre los pacientes pre-hipertensos. En el estudio de Egert et al., ${ }^{27}$ los pacientes con síndrome metabólico fueron clasificados de acuerdo con sus fenotipos ApoE. La quercetina redujo la presión arterial sistólica en el grupo ApoE3, mientras que no fueron observados efectos significativos en los pacientes con el fenotipo ApoE4. En voluntarios sanos, ni el consumo crónico de quercetina, ${ }^{28}$ ni su administración aguda ${ }^{29}$ modificaron significativamente algunos factores de riesgo cardiovascular incluyendo la presión arterial. No obstante, esta falta de efecto en los sujetos sanos, no es sorprendente dada la falta de efecto de la quercetina en animales sanos como se ha descrito anteriormente.

\section{Enigma farmacocinético: La paradoja de los flavonoides}

Los flavonoides se ingieren regularmente en la dieta en forma de mezclas complejas de diferentes compuestos tipo flavonoide junto a otras sustancias activas. Después de la ingestión oral, los flavonoides están sujetos a modificación química dentro del tracto gastrointestinal por las enzimas del hospedador y de la microbiota y después de la absorción son metabolizados en la pared intestinal, el hígado y los tejidos periféricos. ${ }^{30}$ La matriz de la comida también puede influir en el metabolismo gastrointestinal y en la absorción. Las concentraciones alcanzadas para la mayoría de estas sustancias están por debajo del rango micromolar y permanecen sin ser detectados por técnicas analíticas comunes, por lo que sólo un pequeño número de compuestos han captado la atención de los científicos. Sin embargo, las bajas concentraciones pueden no indicar necesariamente falta de actividad biológica de una sustancia muy potente. Aquí nos centraremos en quercetina, uno de los flavonoides más ampliamente distribuidos en el dieta.

Los primeros estudios sobre la farmacocinética de la quercetina en seres humanos ${ }^{31}$ encontraron que la absorción oral de la quercetina era mínima y no se pudieron detectar concentraciones cuantificables en plasma $\mathrm{u}$ orina $\mathrm{y}$, por lo tanto, los autores concluyeron que la administración oral de flavonoides pueden ser de valor biológico cuestionable. Estudios posteriores confirmaron que la quercetina como aglicona fue indetectable en plasma. ${ }^{32}$ Paradójicamente, un 
gran número de estudios en humanos y animales muestran que la administración oral de quercetina ejerce efectos biológicos sistémicos claros. ${ }^{1,33}$ Por lo tanto, la paradoja de flavonoides puede ser descrita de la siguiente manera: A pesar de que la quercetina no se encuentra en el plasma después de la administración oral, ejerce efectos sistémicos demostrables.

En la década pasada quedó claro que aunque la quercetina estaba ausente en el plasma como una aglicona, estaba presente en forma conjugada con ácido glucurónico o sulfato. La quercetina puede administrarse por vía oral como una aglicona en los complementos alimenticios o se ingiere como formas glicosiladas en la dieta. Los glicósidos son hidrolizados por la boca y las bacterias intestinales y por la lactasa-floricina hidrolasa del borde en cepillo de las células epiteliales, pero algunos pueden ser también absorbidos intactos a través del transportador de glucosa SGLT1 y después hidrolizados intracelularmente. La quercetina es rápidamente conjugada con ácido glucurónico y/o sulfato durante el metabolismo de primer paso en la pared intestinal o en el hígado, y una parte de los metabolitos también pueden ser metilados. Las enzimas responsables para estos procesos metabólicos son UDP-glucuronosiltransferasas, sulfotransferasas y metiltransferasas y las principales metabolitos de quercetina en plasma ${ }^{32}$ humano son quercetina-3-glucurónido, quercetina-3'-sulfato e isorhamnetina3-glucurónido (3'-metilquercetina-3-glucurónido) (Figura 2).

Figura 2. Metabolitos plasmáticos de quercetina
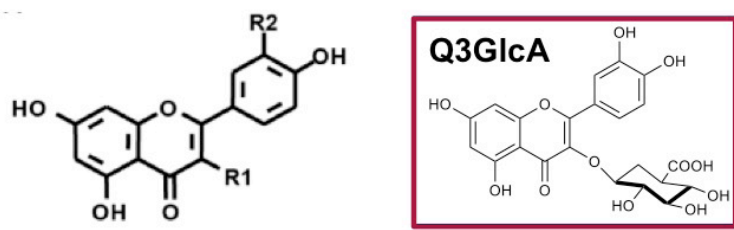

\begin{tabular}{|l|l|l|}
\hline & \multicolumn{1}{|c|}{ R1 } & \multicolumn{1}{c|}{ R2 } \\
\hline Quercetina & $\mathrm{OH}$ & $\mathrm{OH}$ \\
\hline Isorhamnetina & $\mathrm{OH}$ & $\mathrm{OCH}_{3}$ \\
\hline $\begin{array}{l}\text { Quercetina-3-glucuronido } \\
\text { (Q3GlcA) }\end{array}$ & $\begin{array}{l}\text { O-ácido } \\
\text { glucurónico }\end{array}$ & $\mathrm{OH}$ \\
\hline $\begin{array}{l}\text { Isorhamnetina-3-glucurónido } \\
\text { (13GIcA) }\end{array}$ & $\begin{array}{l}\text { O-ácido } \\
\text { glucurónico }\end{array}$ & $\mathrm{OCH}_{3}$ \\
\hline Quercetina-3'-sulfato (Q3'S) & $\mathrm{OH}$ & $\mathrm{OSO}_{3} \mathrm{H}$ \\
\hline
\end{tabular}

Durante décadas se ha generado una enorme cantidad de datos científicos de estudios in vitro con la aglicona quercetina y otros flavonoides relacionados. Debido a que la quercetina no puede ser encontrada en el plasma, la relevancia de todos estos estudios con la aglicona ha sido cuestionados. ${ }^{34}$ Por tanto, se propuso que los estudios mecánicos realizados in vitro deberían repetirse con las principales formas conjugadas que se encuentran en el plasma. Estos estudios se han retrasado durante varios años por la falta de compuestos disponibles comercialmente. La información acerca de los efectos in vitro de estos metabolitos indica que son menos activos que el compuesto del que derivan y a menudo son totalmente inactivos. Por ejemplo, en contraste con las agliconas, ${ }^{35}$ los metabolitos glucuronizados y sulfatados carecen de efecto vasodilatador agudo directo en arterias aisladas y tienen sólo un efecto parcial en la prevención de la disfunción endotelial aguda. ${ }^{36}$ Por lo tanto, la sugerencia de que los metabolitos conjugados son la formas activas responsables de los efectos biológicos in vivo no está totalmente apoyada por la evidencia actual. Así, la paradoja de los flavonoides podría ser actualizada como sigue: Quercetina oral ejerce efectos sistémicos demostrables, pero sus formas circulantes en el plasma después de la administración oral muestran una débil actividad in vitro.

A pesar de que las agliconas quercetina e isorhamnetina no se han descubierto en el plasma, están presentes en cantidades considerables en los tejidos, junto con cantidades variables de metabolitos conjugados. ${ }^{37}$ Además, los metabolitos glucurono-conjugados de quercetina pueden ser hidrolizados por $\beta$-glucuronidasas, liberando la aglicona correspondiente. ${ }^{38}$ La hidrólisis de los glucurónidos de flavonoides fue demostrada por y Shimoi et al. ${ }^{39}$ en neutrófilos humanos y por Lee-Hilz et al. ${ }^{40}$ en el líneas celulares de carcinoma. Estos autores sugirieron que la actividad de los metabolitos del flavonoide depende de su desconjugación in situ. Kawai et al., ${ }^{41}$ sobre la base de estas y otras evidencias indirectas, propuso que los glucurónidos conjugados de quercetina funcionan no sólo como metabolitos de desintoxicación sino también como precursores de la aglicona hidrofóbica bioactiva. $^{42}$

Para entender la paradoja de los flavonoides nos planteamos algunos preguntas fundamentales: ${ }^{43}$

1. ¿Los glucurónidos de quercetina son activos in vivo? Para abordar esta tema, se inyectó la quercetina-3-glucurónido por vía intravenosa $\left(1 \mathrm{mg} \mathrm{kg}^{-1}\right)$ en ratas (es decir, directamente en la sangre, donde se encuentra después de la administración oral de quercetina), y se controló continuamente la presión arterial. Se debe tener en cuenta que este metabolito era totalmente inactivo como vasodilatador agudo in vitro, tanto en arterias de conducción ${ }^{36}$ como en pequeñas arterias de resistencia (datos no publicados). Curiosamente, in vivo, quercetina-3-glucurónido produjo una disminución progresiva de la presión arterial a través del tiempo. Así, el glucurónido en el plasma era capaz de imitar los efectos de la quercetina por vía oral, lo que sugiere que el glucurónido podría ser responsable de los efectos de las agliconas. Sin embargo, debemos señalar que el efecto 
no fue inmediato; era significativo sólo 2 h después de su administración, y fue más persistentes (casi 8 h) que la presencia del compuesto en el plasma.

2. ¿Los glucurónidos de quercetina son desconjugados in vitro en los tejidos diana? Para su estudio se utilizó el lecho mesentérico aislado y perfundido como un modelo vascular en el que el glucurónido era continuamente recirculado con la ayuda de una bomba peristáltica. ${ }^{43}$ La aglicona apareció lentamente en la solución recirculada (detectable después 1 o 2 h), de forma paralela con la disminución de la concentración glucurónido. Las agliconas pudieron ser también encontradas en el tejido a las 3 y 6 h. Estos datos indican que quercetina-3-glucurónido se puede desconjugar en la vascular pared. Por otra parte, se pudo medir la expresión y la actividad de la enzima $\beta$-glucuronidasa en la pared vascular.

3. ¿Los conjugados ejercen efectos in vitro cuando se incuban durante tiempo suficiente para ser desconjugados? Cuando un anillo arterial mesentérico aislado se incubó con quercetina-3-glucurónido durante 3 horas la vasoconstricción inducida por fenilefrina fue inhibida, mientras que la incubación durante 30 min no tuvo efecto. ${ }^{15}$ En células musculares lisas vasculares (CMLV) procedentes de aorta de ratas WKY, la incubación durante 3 horas con quercetina-3-glucurónido inhibió la actividad NADPH oxidasa a dosis que no produjeron efecto después de 30 min de incubación. $^{44}$

4. ¿La inhibición de la desconjugación previene los efectos de la los glucurónidos? Se utilizó el inhibidor clásico de $\beta$-glucuronidasa, sacarolactona, que había sido previamente encontrado como inhibidor de la desconjugación de quercetina-glucurónido en extractos de hígado. ${ }^{38}$ Esta fármaco redujo la actividad $\beta$-glucuronidasa en la pared vascular, lo que se acompañó con la inhibición de la desconjugación en el lecho vascular mesentérico perfundido y de los efectos a largo plazo de glucurónidos en el arteria mesentérica aislada ${ }^{43}$ y en CMLV. ${ }^{44}$ Todos estos resultados apoyan que los glucurónidos circulantes se comportan plasma como portadores de quercetina aglicona y que la quercetina liberada en los órganos diana tras desconjugación es la efectora final.

Se estudió además la paradoja de los flavonoides en un modelo animal de la enfermedad: la rata espontáneamente hipertensas (SHR). ${ }^{45}$ Se ha encontrado que los efectos reductores de la presión arterial cuando se administra por vía intravenosa quercetina-3-glucuronido son dependientes de la dosis; este metabolito fue eficaz a dosis tan bajas como 0,2 $\mathrm{mg} \mathrm{kg}$. . Isorhamnetin-3-glucurónido era también eficaz in vivo, mientras que el tercer metabolito principal en plasma, quercetina-3'-sulfato, no mostró efecto. Estos efectos in vivo de quercetina-3-glucurónido se prevenían mediante la inhibición de la desconjugación con sacarolactona. Finalmente, se analizó si el proceso de desconjugación tenía un papel fisiológico, es decir, si estaba involucrado en los efectos de la quercetina cuando se administra por vía oral. De hecho, la inhibición de la $\beta$-glucuronidasa con sacarolactona abolió los efectos antihipertensivos de quercetina oral. ${ }^{16}$ Además, después de $5 \mathrm{~h}$ de la administración oral de quercetina en voluntarios sanos se observó un aumento del diámetro de la arteria braquial que se correlaciona con el producto de los niveles plasmáticos de quercetina-3-glucurónido por la actividad glucuronidasa medida a las $2 \mathrm{~h} .{ }^{29}$ Estos resultados indican que la conjugación y posterior desconjugación son procesos necesarios para los efectos de la quercetina administrada por vía oral.

En conjunto, la evidencias disponibles actualmente indican que quercetina oral se absorbe parcialmente en el intestino; es ampliamente metabolizada en metabolitos glucurono- $y$ sulfo-conjugados, que son las formas circulantes en plasma, y glucurono-, pero no sulfo-conjugados, pueden ser hidrolizados en el vascular nivel, produciendo la aglicona, que puede ser acumulada en los tejidos. Por lo tanto, la conjugación es un proceso reversible y, al menos en relación con los efectos vasodilatadores y antihipertensivos, el ciclo de conjugación-deconjugation parece ser un requisito absoluto. Los derivados glucuronizados transportan quercetina y liberan la aglicona libre in situ por desconjugación. La desconjugación parece ser un proceso más lento que la conjugación. Como la quercetina es más lipófila que los metabolitos puede permanecer atrapada en los tejidos, interactuar con bicapas de fosfolípidos en membranas celulares y subcelulares, y cualquier quercetina liberada de los tejidos pueden ser re-conjugada de nuevo en el hígado, lo que explica sus bajos niveles plasmáticos. Sin embargo, algunos efectos pueden ser atribuidos a los metabolitos conjugados por sí mismos y queda por demostrar si la desconjugación también es necesaria para las otras importantes acciones de los flavonoides, especialmente los efectos antiaterogénicos. Además, el papel de otros metabolitos, en particular, aquellos producidos por la microbiota intestinal que también pueden entrar en la circulación sistémica, debe ser estudiado. ${ }^{46}$

Algunas consideraciones prácticas surgen de lo anterior. La más importante es que, debido a que la quercetina parece ser el efector final, los cientos de estudios mecanísticos que se han llevado a cabo con quercetina aglicona in vitro no son inútiles. En segundo lugar, en los estudios que analizan los efectos de los metabolitos conjugados in vitro, la presencia y la actividad de $\beta$-glucuronidasa en el tipo ce- 
lular estudiado puede ser esencial y el factor tiempo debe ser también tenido en cuenta, porque la desconjugación es un proceso lento. En tercer lugar, porque la pared del vaso es capaz de desconjugar glucurónidos y estos metabolitos deben cruzar esta barrera para llegar a cualquier tejido, se espera que la aglicona puede entrar en los tejidos de forma independiente de la actividad $\beta$-glucuronidasa de las células no vasculares en el órgano. De hecho, en todos los tejidos analizados la aglicona se encontró pero la actividad $\beta$-glucuronidasa específica no se correlacionó con las proporciones de flavonoles deconjugados en los distintos tejidos. ${ }^{37}$ Cuarto, sacarolactona es una herramienta útil para analizar el papel del proceso de conjugación-deconjugación tanto in vivo como in vitro. En quinto lugar, la actividad $\beta$-glucuronidasa puede influir en la eficacia de quercetina y ésto puede ser determinado genéticamente. Por ejemplo, la actividad $\beta$-glucuronidasa fue mayor en ratones transgénicos que expresan apoE3 en comparación con aquellos que expresan apoE $4,{ }^{47}$ lo que puede estar relacionado con la disminución de la presión arterial sistólica inducida por quercetina en patientes con un genotipo apoE3, mientras que no tiene efecto significativo en el grupo de apoE4. Además, la actividad $\beta$-glucuronidasa se incrementa por endotoxina bacteriana, ${ }^{39}$ lo que sugiere que la quercetina puede ser más activa en condiciones inflamatorias, tales como en la aorta humana lesionada con placas ateroscleróticas. ${ }^{41}$ Finalmente, eficacias catalíticas similares se obtuvieron para la desconjugación de O-glucurónidos de quercetina sustituidos en diferente posiciones ${ }^{48}$ y para la desconjugación de otros flavonoles e isoflavonoides, ${ }^{38}$ lo que sugiriere que todo lo expuesto anteriormente puede ser válido para flavonoides diferentes de quercetina.

\section{CONCLUSIONES}

Los metabolitos glucurono-conjugados transportan en el plasma la quercetina y su forma metilada, y liberan en los tejidos la aglicona libre, que es el efector final de sus efectos biológicos (Figura 3).

Figura 3. Paradoja de los flavonoides

\section{Quercetina}

conjugación intestinal o hepática

Metabolitos inactivos o menos activos desconjugación local

\section{Quercetina}

\section{Efectos in vivo}

\section{REFERENCIAS}

1. Perez-Vizcaino F, Duarte J, Flavonols and cardiovascular disease. Mol Aspects Med. 2010;31(6):478-494.

2. Martínez-Flórez S, González-Gallego J, Culebras JM, Tuñón MJ. Flavonoids: properties and anti-oxidizing action. Nutr Hosp. 2002;17(6):271-278.

3. Hertog MG, Feskens EJ, Hollman PC, Katan MB, Kromhout D. Dietary antioxidant flavonoids and risk of coronary heart disease: the Zutphen Elderly Study. Lancet. 1993;342(8878):10071011.

4. Manach C, Scalbert A, Morand C, Remesy C, Jiménez L. Polyphenols: food sources and bioavailability. Am J Clin Nutr. 2004;79(5):727-747.

5. Geleijnse JM, Launer LJ, Van der Kuip DA, Hofman A, Witteman JC. Inverse association of tea and flavonoid intakes with incident myocardial infarction: the Rotterdam Study. Am J Clin Nutr. 2002;75(5):880-886.

6. Hertog MG, Bueno-de-Mesquita HB, Fehily AM, Sweetnam PM, Elwood PC, Kromhout D. Fruit and vegetable consumption and cancer mortality in the Caerphilly Study. Cancer Epidemiol Biomarkers Prev. 1996;5(9):673-677.

7. Huxley RR, Neil HA. The relation between dietary flavonol intake and coronary heart disease mortality: a meta-analysis of prospective cohort studies. Eur J Clin Nutr. 2003;57(8):904908.

8. Miedema MD, Petrone A, Shikany JM, Greenland P, Lewis CE, Pletcher MJ, et al. The Association of Fruit and Vegetable Consumption During Early Adulthood With the Prevalence of Coronary Artery Calcium After 20 Years of Follow-Up: The CARDIA Study. Circulation. 2015. pii: CIRCULATIONAHA.114.012562.

9. Duarte J, Perez-Vizcaino F, Utrilla P, Jimenez J, Tamargo J, Zarzuelo A. Vasodilatory effects of flavonoids in rat aortic smooth muscle. Structure-activity relationships. Gen Pharmacol. 1993;24(4):857-862.

10. Carlstrom J, Symons JD, Wu TC, Bruno RS, Litwin SE, Jalili T. A quercetin supplemented diet does not prevent cardiovascular complications in spontaneously hypertensive rats. J Nutr. 2007;137(3):628-633.

11. Machha A, Mustafa MR. Chronic treatment with flavonoids prevents endothelial dysfunction in spontaneously hypertensive rat aorta. J Cardiovasc Pharmacol. 2005;46(1):36-40.

12. Romero M, Jimenez R, Sanchez M, Lopez-Sepulveda R, Zarzuelo MJ, O’Valle F, et al. Quercetin inhibits vascular superoxide production induced by endothelin-1: Role of NADPH oxidase, uncoupled eNOS and PKC. Atherosclerosis. 2009;202(1):58-67.

13. Sanchez M, Galisteo M, Vera R, Villar IC, Zarzuelo A, Tamargo $\mathrm{J}$, et al. Quercetin downregulates NADPH oxidase, increases 
eNOS activity and prevents endothelial dysfunction in spontaneously hypertensive rats. J Hypertens. 2006;24(1),75-84.

14. Duarte J, Jimenez R, O'Valle F, Galisteo M, Perez-Palencia $\mathrm{R}$, Vargas $\mathrm{F}$, et al. Protective effects of the flavonoid quercetin in chronic nitric oxide deficient rats. J Hypertens. 2002;20(9):1843-1854.

15. Galisteo M, Garcia-Saura MF, Jimenez R, Villar IC, Wangensteen R, Zarzuelo A, et al. Effects of quercetin treatment on vascular function in deoxycorticosterone acetate-salt hypertensive rats. Comparative study with verapamil. Planta Med. 2004;70(4):334-341.

16. Garcia-Saura MF, Galisteo M, Villar IC, Bermejo A, Zarzuelo A, Vargas F, et al. Effects of chronic quercetin treatment in experimental renovascular hypertension. Mol Cell Biochem. 2005;270(1-2):147-155.

17. Aoi W, Niisato N, Miyazaki H, Marunaka Y. Flavonoid-induced reduction of ENaC expression in the kidney of Dahl saltsensitive hypertensive rat. Biochem Biophys Res Commun. 2004;315(4):892-896.

18. Mackraj I, Govender T, Ramesar S. The antihypertensive effects of quercetin in a salt-sensitive model of hypertension. J Cardiovasc Pharmacol. 2008;51(3):239-245.

19. Jalili T, Carlstrom J, Kim S, Freeman D, Jin H, Wu TC, et al. Quercetin-supplemented diets lower blood pressure and attenuate cardiac hypertrophy in rats with aortic constriction. J Cardiovasc Pharmacol. 2006;47(4):531-541.

20. Rivera L, Moron R, Sanchez M, Zarzuelo A, Galisteo M. Quercetin ameliorates metabolic syndrome and improves the inflammatory status in obese Zucker rats. Obesity (Silver Spring). 2008;16(9):2081-2087.

21. Yamamoto $Y$, Oue E. Antihypertensive effect of quercetin in rats fed with a high-fat high-sucrose diet. Biosci Biotechnol Biochem. 2006;70(4):933-939.

22. Barker DJ. In utero programming of chronic disease. Clin Sci (Lond). 1998;95(2):115-28.

23. Buckley AJ, Keseru B, Briody J, Thompson M, Ozanne SE, Thompson $\mathrm{CH}$. Altered body composition and metabolism in the male offspring of high fat-fed rats. Metabolism. 2005;54(4):500-507.

24. Liang C, Oest ME, Prater MR. Intrauterine exposure to high saturated fat diet elevates risk of adult-onset chronic diseases in C57BL/ 6 mice. Birth Defects Res B Dev Reprod Toxicol. 2009;86(5):377-384.

25. Turnbull F. Effects of different blood-pressure-lowering regimens on major cardiovascular events: results of prospectively-designed overviews of randomised trials. Lancet. 2003;362(9395):1527-1535.
26. Edwards RL, Lyon T, Litwin SE, Rabovsky A, Symons JD, Jalili T. Quercetin reduces blood pressure in hypertensive subjects. J Nutr. 2007;137(11):2405-2411.

27. Egert S, Boesch-Saadatmandi C, Wolffram S, Rimbach G, Muller MJ. Serum lipid and blood pressure responses to quercetin vary in overweight patients by apolipoprotein E genotype. J Nutr. 2010;140(2):278-284.

28. Conquer JA, Maiani G, Azzini E, Raguzzini A, Holub BJ. Supplementation with quercetin markedly increases plasma quercetin concentration without effect on selected risk factors for heart disease in healthy subjects. J Nutr. 1998;128(3):593597.

29. Perez A, Gonzalez-Manzano S, Jimenez R, Perez-Abud R, Haro JM, Osuna A, et al. The flavonoid quercetin induces acute vasodilator effects in healthy volunteers: correlation with beta-glucuronidase activity. Pharmacol Res. 2014;89:11-8.

30. Manach C, Williamson G, Morand C, Scalbert A, Remesy C. Bioavailability and bioefficacy of polyphenols in humans. I. Review of 97 bioavailability studies. Am J Clin Nutr. 2005;81(1 Suppl):230S-242S.

31. Gugler R, Leschik M, Dengler HJ. Disposition of quercetin in man after single oral and intravenous doses. Eur J Clin Pharmacol. 1975;9(2-3):229-234.

32. Day AJ, Mellon F, Barron D, Sarrazin G, Morgan MR, Williamson G. Human metabolism of dietary flavonoids: identification of plasma metabolites of quercetin. Free Radic Res. 2001;35(6):941-952.

33. Williamson G, Manach C. Bioavailability and bioefficacy of polyphenols in humans. II. Review of 93 intervention studies. Am J Clin Nutr. 2005;81(1 Suppl):243S-255S.

34. Kroon PA, Clifford MN, Crozier A, Day AJ, Donovan JL, Manach $C$, et al. How should we assess the effects of exposure to dietary polyphenols in vitro? Am J Clin Nutr. 2004;80(1):1521.

35. Perez-Vizcaino F, Ibarra M, Cogolludo AL, Duarte J, Zaragoza-Arnaez F, Moreno L, et al. Endothelium-independent vasodilator effects of the flavonoid quercetin and its methylated metabolites in rat conductance and resistance arteries. J Pharmacol Exp Ther. 2002;302(1):66-72.

36. Lodi F, Jimenez R, Moreno L, Kroon PA, Needs PW, Hughes DA, et al. Glucuronidated and sulfated metabolites of the flavonoid quercetin prevent endothelial dysfunction but lack direct vasorelaxant effects in rat aorta. Atherosclerosis. 2009;204(1):34-39.

37. Bieger J, Cermak R, Blank R, de Boer VC, Hollman PC, Kamphues J, et al. Tissue distribution of quercetin in pigs after long-term dietary supplementation. J Nutr. 2008;138(8):14171420. 
38. O'Leary KA, Day AJ, Needs PW, Sly WS, O'Brien NM, Williamson $\mathrm{G}$, Flavonoid glucuronides are substrates for human liver beta-glucuronidase. FEBS Lett. 2001;503(1):103-106.

39. Shimoi K, Saka N, Nozawa R, Sato M, Amano I, Nakayama $\mathrm{T}$, et al. Deglucuronidation of a flavonoid, luteolin monoglucuronide, during inflammation. Drug Metab Dispos. 2001;29(12):1521-1524.

40. Lee-Hilz YY, Stolaki M, van Berkel WJ, Aarts JM, Rietjens IM. Activation of EpRE-mediated gene transcription by quercetin glucuronides depends on their deconjugation. Food Chem Toxicol. 2008;46(6):2128-2134.

41. Kawai Y, Nishikawa T, Shiba Y, Saito S, Murota K, Shibata $\mathrm{N}$, et al. Macrophage as a target of quercetin glucuronides in human atherosclerotic arteries: implication in the anti-atherosclerotic mechanism of dietary flavonoids. J Biol Chem. 2008;283(14):9424-9434.

42. Terao J, Murota K, Kawai Y, Conjugated quercetin glucuronides as bioactive metabolites and precursors of aglycone in vivo. Food Funct. 2011;2(1):11-17.

43. Menendez C, Dueñas M, Galindo P, Gonzalez-Manzano S, Jimenez R, Moreno L, et al. Vascular deconjugation of quercetin glucuronide: The flavonoid paradox revealed? Mol Nutr Food Res. 2011;55(12):1780-1790.
44. Jimenez R, Lopez-Sepulveda R, Romero M, Toral M, Cogolludo A, Perez-Vizcaino F, et al. Quercetin and its metabolites inhibit the membrane NADPH oxidase activity in vascular smooth muscle cells from normotensive and spontaneously hypertensive rats. Food Funct. 2015;6(2):409-414.

45. Galindo P, Rodriguez-Gómez I, González-Manzano S, Dueñas M, Jiménez R, Menéndez C, et al. Glucuronidated quercetin lowers blood pressure in spontaneously hypertensive rats via deconjugation. PLoS One. 2012;7(3):e32673.

46. van Duynhoven J, Vaughan EE, Jacobs DM, Kemperman RA, van Velzen EJ, Gross G, et al. Metabolic fate of polyphenols in the human superorganism. Proc Natl Acad Sci U S A. 2011;108(Suppl 1):4531-4538.

47. Boesch-Saadatmandi C, Niering J, Minihane AM, Wiswedel I, Gardeman A, Wolffram S. Impact of apolipoprotein E genotype and dietary quercetin on paraoxonase 1 status in apoE3 and apoE4 transgenic mice. Atherosclerosis. 2010;211(1):110113.

48. Bartholome R, Haenen G, Hollman CH, Bast A, Dagnelie PC, Roos D, et al. Deconjugation kinetics of glucuronidated phase II flavonoid metabolites by beta-glucuronidase from neutrophils. Drug Metab Pharmacokinet. 2010;25(4):379-387. 